\title{
L'éthique de la communication : une politique des droits
} démocratiques?

In: Quaderni. N. 28, Hiver 1996. pp. 147-156.

Citer ce document / Cite this document :

Chanial Philippe. L'éthique de la communication : une politique des droits démocratiques ?. In: Quaderni. N. 28, Hiver 1996. pp. 147-156.

doi : 10.3406/quad.1996.1557

http://www.persee.fr/web/revues/home/prescript/article/quad_0987-1381_1996_num_28_1_1557 


\title{
L'ÉTHIQUE DE LA COMMUNICATION : UNE POLITIQUE DES DROITS DÉMOCRATIQUES?
}

\author{
PHILIPPE CHANIAL
}

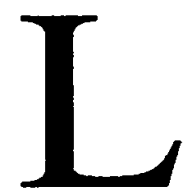

a théorie de la communication peut-elle instruire d'une quelconque façon la théorie démocratique?

Dans les plus récents développements de son oeuvre, J.Habermas tente de relever un tel pari. Il s'agit néanmoins d'un pari risqué tant il peut autoriser de multiples formes de dissolution naïve, voire idéologique, de la politique démocratique dans la communication et ses vertus consensuelles.

C'est pour tenter de conjurer une telle aporie qui menace parfois les analyses de Habermas et plus souvent celles de ses commentateurs, que nous voudrions, dans cet article, proposer une lecture politique de l'éthique de la communication du sociologue allemand.

Ce projet rencontre immédiatement des résistances de l'intérieur même du texte d'Habermas. En effet, dans la généalogie de l'agir communicationnel (1987a, 1987b), le dévoilement de ses implications morales et l'exposé des principes de l'éthique de la discussion (1986, 1992), la question de la démocratie n'apparait qu'en creux. L'horizon de la démocratie est certes présupposé dans le concept même de "communauté de communication idéale", ou plus généralement dans celui même

Maître de conférence LASAR - Université de Caen

Errata : Dans notre numéro 26, des erreurs de mise en page ont malencontreusement altéré cet article, que nous redonnons dans sa version corrigée. d'intersubjectivité. L'exigence démocratique semble ainsi participer d'un mouvement historique et constituer un acquis de la rationalisation moderne grâce auquel se dévoile et prend forme un potentiel de rationalité inscrit dans les structures mêmes de l'intersubjectivité pratique. L'éthique de la discussion (1) se présente alors comme la simple formalisation des implications éthiques inscrites au coeur même des structures formelles de l'agir communicationnel et, in fine, la démocratie comme la mise en 
forme politique de cette éthique discursive.

Il est ainsi tentant de déduire de cette éthique communicationnelle les conditions d'une politique démocratique, c'est à dire à la fois les modalités d'une formation libre de l'opinion et de la volonté démocratique et les critères de justification et de critique des normes, institutions et décisions dans l'espace démocratique. Cette déduction mécanique, rapportant ultimement le politique à l'éthique, Habermas y succombe parfois. Telle est toute l'ambiguité du projet habermassien. Comme le notent J.Cohen et A.Arato : "J.Habermas considère d'une part l'éthique de la discussion comme une théorie morale universaliste, dans la tradition kantienne. D'autre part, il la présente également comme le coeur d'une théorie de la légitimité démocratique, offrant une alternatives aux théories traditionnelles et néo-contractualistes" (1992 : 350).

Cette ambiguité oblige, si l'on souhaite s'en dégager tout en préservant les acquis de la démarche habermassienne, de construire une lecture politique de l'éthique de la discussion, sensible à ce qu'élude une perspective éthique, à savoir les médiations institutionnelles constitutives du fait politique dans les démocraties modernes. Ainsi, cette interprétation exige moins de pointer en quoi la visée d'un consensus rationnellement motivé constituerait l'idéal en acte d'un ordre politique démocratique que de montrer en quoi l'éthique de la communication est indissociable d'une politique des droits démocratiques.

Si cette éthique politique est inscrite dans le projet même de la modernité démocratique, c'est avant tout, nous tenterons de le montrer, parce qu'elle présuppose un système de droits fondamentaux, et cela dans un double sens. D'une part, en amont, pourrait-on dire, cette éthique discursive exige la reconnaissance de tels droits démocratiques comme condition de sa visée pratique. D'autre part, en aval, parce que son projet est celui d'une démocratisation toujours plus radicale de la société, l'éthique de la discussion implique, comme horizon critique, l'approfondissement et l'élargissement de ces droits. L'argument politique de Habermas serait peut-être alors celui-ci : si la réalisation de la démocratie exige une extension toujours inachevée et toujours menacée de la discussion publique à un réseau sans cesse plus large de relations sociales, ce projet, parce qu'il doit faire face à la réalité des rapports de pouvoir, à la dynamique de répression systématique des intérêts universalisables, doit bénéficier de garanties institutionnelles - un système de droits fondamentaux - tout en en visant la généralisation continue comme la réinterprétation critique.

\section{LES DEUX FACES DU DROIT RATION- NEL MODERNE}

La théorie des droits du sociologue allemand repose sur une double conceptualisation, révélatrice des ambivalences du droit moderne (1987a, 1987b). Cette ambivalence, Habermas la rapporte au double processus au coeur de la rationalisation occidentale : sa dimension instrumentale et sa dimension morale-pratique.

Sous ce premier aspect, le droit ne constitue qu'un médium, au sens de T.Parsons, propre à remplir les impératifs fonctionnels d'un système politique bureaucratique et d'un système économique capitaliste. Ce premier type de droit, le droit-médium, renvoie donc à la face instrumentale, systémique du processus de ra- 
tionalisation. Y prime l'efficacité fonctionnelle. Sa validité n'est soumise qu'au principe de légalité. Habermas note ainsi que "le droit appliqué comme médium se débarasse de la problématique de la légitimation", car il n'est relié au corpus juridique "uniquement par des procédures formellement correctes". Cependant si le droit comme médium peut être déchargé de toute exigence de justification, la référence au principe de légalité - central dans la sociologie du droit de Max Weber - ne suffit pas à assurer la validité de toutes les normes juridiques.

La seconde forme du droit moderne, le droitinstitution, rassemble justement ces normes qui ne sauraient trouver de fondement dans le simple renvoi positivistes à des procédures formelles. Ce concept de droit-institution permet ainsi à l'auteur de sortir du cercle vicieux dans lequel la croyance en la légalité ne peut engendrer la légitimité que si la légitimité de l'ordre juridique est déjà présupposée (2). Pour que la légalité d'une décision soit l'indice de la légitimité, il faut que cet ordre juridique lui-même repose sur des fondements rationnels, c'est à dire sur des principes qui, à la différence des simples décisions, exigent une justification substantielle. Cette justification ressort, selon Habermas, d'une morale fondée sur des principes : "Le catalogue des droits fondamentaux que contiennent les constitutions bourgeoises (...) exprime la nécessité structurelle de la justification à côté du principe fondamentale de la souveraineté populaire, liant la compétence législative à l'accord d'une volonté démocratiquement formée" (1987a : 272). Ces principes, qu'ils soient d'ailleurs consignés ou non dans des textes formels, ont pour particularité de ne pas dépendre du droit positif puisqu'ils en constituent l'instance de légitimation, puisqu'ils prétendent fonder la légitimité de la légalité. La politique démocratique présuppose donc ces deux piliers normatifs : souveraineté populaire et droits fondamentaux. Néanmoins, en quoi l'éthique de la discussion se rapporte-telle à de tels principes ?

\section{DE LA THÉORIE DES DROITS DÉMO- CRATIQUES À L'ÉTHIQUE DE LA DIS- CUSSION}

L'argument proposé par Habermas, afin de lier son éthique de la communication et les exigences d'une politique des droits démocratiques, peut être ainsi reconstruit : la procédure même de l'argumentation qui soutient l'éthique de la discussion est indissociable d'un ensemble de "valeurs", elle n'est donc pas purement formelle. Et ces "valeurs" peuvent être analysées comme l'expression même des principes démocratiques, en tant qu'ils constituent les conditions d'une "intersubjectivité authentique". Bref, l'éthique de la discussion implique la reconnaissance de droits fondamentaux.

L'alternative que propose Habermas se distingue ainsi radicalement de celle élaborée par John Rawls (1987). Cette dernière, il suffit de le rappeler, consiste à fonder ces principes fondamentaux - en l'occurence deux grands principes de justice - à partir d'une procédure de délibération collective fictive : la position originelle sous le "voile d'ignorance". Le procéduralisme de Habermas s'oppose à celui de Rawls : les principes démocratiques selon le philosophe allemand ne saurait être déduits - ou dévoilés - à partir d'une procédure de choix rationnel. En effet, le résultat (les principes retenus) d'une telle procédure, lorsqu'elle est envisagée non comme une expérience de pensée fictive mais comme une délibération collective effective, est toujours ouvert, n'est jamais définitif. 
Habermas propose en fait un raisonnement inverse : si les principes ne sont pas, pour une partie d'entre eux au moins, postérieurs à la procédure délibérative, ils ne peuvent être qu'antérieurs à celle-ci. Ou plus précisément, ils doivent être incarnés dans cette procédure même. C'est cette hypothèse de lecture, qui nous semble implicite dans le texte de Habermas, notamment dans son interprétation de l'analyse de Fröber (Habermas 1989), que nous nous proposons de construire dans le prolongement de la réflexion de quelques commentateurs de l'auteur.

\section{LES PRÉSUPPOSITIONS SUBSTAN- TIELLES DE L'ÉTHIQUE DE LA DIS- CUSSION.}

Notre première démonstration s'appuiera sur les analyses de S.Benhabib $(1986,1990)$ qui tente de reconstruire l'argument habermassien en dévoilant ses présuppositions substantielles.

Selon la philosophe américaine, l'éthique de la discussion de Habermas est menacée d'un double écueil : la trivialité et l'inconsistance. Si le modèle de la discussion pratique est défini de façon minimale, à l'image par exemple du rationalisme critique d'un Popper, il est trivial dans ses implications : est juste, bon... ce qui a fait l'objet d'un accord intersubjectif. Mais d'autre part, il est inconsistant si des présuppositions substantielles, qui n'appartiennent pas aux conditions définissant la situation d'argumentation, viennent nourrir, à l'externe, l'éthique de la discussion. La trivialité conduit ainsi à la circularité (est juste ce qui est (dit) juste), l'inconsistance au dogmatisme (est juste ce qui est conforme à des valeurs étrangères au processus même de l'argumentation). La solution proposée par $S$.Benhabib consiste à mettre en valeur les "affirmations éthiques fortes" que présuppose la situation de parole idéale. L'auteur distingue deux de ces affirmations (1990 : 337-338):

1. Un principe de respect moral universel : il consiste à reconnaître à chaque être humain capable de parole et d'action le droit de participer à la discussion éthique.

2. Un principe de réciprocité égalitaire : selon ce principe, chacun a un droit identique à celui d'autrui d'initier de nouvelles questions, de demander que les présuppositions mêmes de la discussions soient discutées...

Les présuppositions véritables de la situation d'argumentation ont ainsi un contenu normatif qui précède l'argument moral lui-même. Leur dévoilement est indissociable d'une perspective interprétative : "les principes du respect universel et de la réciprocité égalitaire constituent notre clarification philosophique de ce qui constitue le point de vue moral inscrit dans l'horizon normatif de la modernité" (1990 : 339). Ces principes ne constituent donc que l'une des interprétations possible des éléments formels de la compétence des agents moraux au stade post-conventionnel. Dans la perspective de la philosophe américaine, cette formulation du contenu normatif propre à l'éthique de la discussion s'inscrit dans l'indétermination constitutive des "formes de vie démocratiques" de la modernité : elle ne présuppose aucune communauté substantielle et donc ne conduit à aucun dogmatisme. Ces principes ne sont pas importés de l'extérieur : ce sont dans les caractéristiques formelles de la discussion pratique que s'expriment les valeurs et principes constitutifs de nos "formes de vie démocratiques". Elle échappe aussi au risque de circularité 
puisque ces principes peuvent être contestés, mais sous une seule forme, l'argumentation.

Les deux principes que propose S.Benhabib sont la condition même de possibilité d'une discussion morale. C'est la poursuite de la discussion qui exige que nous soutenions des relations de respect universel et de réciprocité égalitaire. En ce sens, l'éthique de la discussion ne constitue pas uniquement une procédure formelle. Elle engage la projection utopique d'une façon de vivre.

\section{LES PRINCIPES DÉMOCRATIQUES, CONDITIONS ET HORIZON DE LA COMMUNICATION DÉMOCRATIQUE.}

Pour convaincant que soit l'argument de S.Benhabib, il ne nous conduit encore qu'imparfaitement vers l'hypothèse que nous visons. Les principes quc formule la philosophie américaine ne renvoient qu'indirectement à ce que nous avons convenu d'appeler les "principes démocratiques". La lecture de deux autres exégètes de Habermas va nous permettre de franchir ce pas supplémentaire : le philosophe allemand A.Wellmer et la philosophe américaine J.Cohen. La question qu'ils posent à Habermas $s$ 'inscrit dans le prolongement de celle adressée par S.Benhabib : la théorie discursive habermassienne est-elle compatible, et sous quelle forme, avec les droits de l'homme et du citoyen, tels que la modernité démocratique les a définis?

\section{ÉTHIQUE DE LA DISCUSSION ET LI- BERTÉ COMMUNAUTAIRE}

Wellmer adresse cette question à Habermas avec une volonté polémique. Wellmer reproche en effet à l'éthique de la discussion non seule- ment son formalisme mais les conséquences de celui-ci en ce qui concerne la reconnaissance des droits individuels. Il place ainsi Habermas face au dilemme hegelien: "Hegel a démontré la futilité d'une conscience morale qui se serait séparée elle-même de la moralité concrète d'une communauté politique ; d'autre part, il démontre également la futilité d'institutions qui compromettraient les droits de la subjectivité"( 1989 : 511). La résolution de ce dilemme par le philosophe d'Iena est bien connue: les droits de la subjectivité ne peuvent servir de supports à l'idée de démocratie comme forme de vie, puisqu'ils ne permettent la constitution d'aucun lien communautaire, d'aucun souci du bien public ; la réconciliation du particulier et de l'universel ne peut s'opérer que dans la morale concrète de l'Etat. Dans les termes de Wellmer, Hegel oppose et subordonne la liberté négative (l'autonomie subjective dans la société civile) à la liberté "communautaire" (notre droit d'auto-détermination en tant que droit politique au sein de la communauté politique, de l'Etat), et annonce ainsi le dilemme même de la Modernité démocratique, déchiré entre l'individualisme et l'esprit de communauté.

C'est à la lumière de ce dilemme que le philosophe allemand rend compte du projet habermassien. Il montre que celui-ci justifie l'exigence de liberté "communautaire" dans son modèle même de rationalité argumentative, dans ses "méta-normes" de la discussion rationnelle, tels qu'elles sont formulées dans les principes de l'éthique de la discussion. Wellmer écrit ainsi :

“La plausibilité de l'idée selon laquelle une conception procédurale de la rationalité, au sens de Habermas, contient déjà une idée de liberté communautaire tient au fait qu'elle définit 
un type post-traditionnel d'accord "éthique" - à savoir un accord sur les méta-normes de l' argumentation rationnelle - et en outre une façon - la seule façon - de rétablir l'accord éthique entre individus libres et égaux, une fois que la substance éthique traditionnelle a été dissoute. A travers la procédure de l'argumentation, la liberté serait liée à la solidarité et à la rationalité ; une conception procédurale de la rationalité définirait ainsi le noyau normatif d'une forme post-traditionnelle de liberté communautaire" (1989 : 537).

La liberté communautaire, ou "positive" au sens d'I.Berlin (1990), est donc intrinsèque à la procédure même de l'argumentation et n'a donc pas à être importée du dehors, elle en constitue une des présuppositions normatives incontournables. Ou plus radicalement encore, les principes formels de l'éthique de la discussion expriment de la façon la plus convaincante cette face de l'exigence moderne de liberté.

Cependant, selon Wellmer, l'autre face de la liberté, la liberté négative, ne saurait être intégrée dans ce modèle de rationalité argumentative. Bien au contraire, ce modèle, comme la figure de l'Etat hegelien, la menace. La démonstration de l'auteur est la suivante : la liberté abstraite, constitutive de l'autonomie du sujet ne peut être déduite d'aucun principe de rationalité puisqu'elle garantit au sujet une sphère d'autonomie dans laquelle il peut exercer librement ses droits, même de façon irrationnelle. Or, "comment un principe de rationalité - même s'il s'agit d'un principe de la rationalité qui régit la "communication" et/ou la "discussion" - pourrait-il dire quoi que ce soit à propos d'un droit de ne pas être rationnel ?"(1989 : 530). Ainsi, "le droit d'être égoïste, fou, excentrique, irresponsable, déviant, ob- sessionnel, auto-destructeur, monomane, etc..."(1989 : 526) est un droit indépendant dont la justification ne peut reposer sur les principes de la rationalité communicationnelle puisque ceux-ci explicitent ce que nous $n$ 'avons pas le droit d'être, les normes que nous n'avons pas le droit de transgresser,...

Il s'agit donc d'un autre type de droit, qui n'est donc ni présupposé dans l'éthique de la discussion, ni susceptible d'être déduit d'une quelconque procédure, justifié par un consensus démocratique rationnel. Sur quoi repose t-il alors ? Sur les "droits de la particularité", de la subjectivité, de la conscience moderne, sur l'héritage libéral des Lumières qu'il faut incorporer, assimiler, comme un corps étranger, dans une conception communautaire de la liberté. En ce sens, Wellmer parait ultimement renouer avec la philosophie de la conscience qu'il prétendait avoir dépassée et refuser toute rupture avec l'argumentaire individualiste.

\section{L'ANCRAGE COMMUNICATIONNEL DES PRINCIPES DÉMOCRATIQUES}

A travers cette analyse de la critique de Wellmer, notre hypothèse initiale s'est vue déjà à moitié validée. Il reste donc à tenter de montrer que mêmes ces droits subjectifs peuvent être justifiées dans le cadre exclusif des conditions et procédures de la discussion rationnelle.

Cette démonstration peut être reconstruite à partir des réflexions de Jean Cohen sur l'éthique de la discussion. Elle initie cette réflexion à partir du dualisme proposé par Wellmer : "Nous avons devant nous deux concepts : la société civile moderne et l'éthique de la discussion. Le premier représente les thèmes du libéralisme classique : le terme "so- 
ciété civile" évoque les droits à l'intimité, à la propriété, à la publicité, à la libre expression, le droit d'association et l'égalité devant la loi. Le second, mettant l'accent sur la participation égale de toutes les personnes concernées à des discussions publiques sur des normes contestées, fait référence aux principes de la démocratie. La vogue actuelle dans la théorie politique contemporaine consiste (une fois encore) à souligner le caractère antithétique du libéralisme et de la démocratie" (1990: 83).

Le projet de J.Cohen consiste donc à dépasser cette antinomie au nom de l'éthique de la discussion, c'est à dire à montrer que celle-ci constitue à la fois une théorie de la légitimité démocratique (justifiant ainsi la revendication de la "liberté communautaire") et une théorie des libertés fondamentales (justifiant de la même façon les exigences de la liberté négative, de l'autonomie subjective). Le coeur de l'argument de la philosophe américaine peut être ainsi résumé. Si l'on comprend l'éthique de la discussion avant tout comme une éthique politique, c'est à dire comme une théorie de la légitimité démocratique et des droits fondamentaux, et non comme une théorie générale du jugement moral, alors elle doit être saisie sous ses dimensions institutionnelles. En effet, elle est indissociable des institutions démocratiques modernes au sens où elle ne peut prétendre à une quelconque effectivité pratique que dans la mesure où elle est déjà, bien que partiellement, incarnée dans un système de droits institutionnalisés.

L'éthique de la discussion appelle une politique des droits démocratiques au double sens où, d'une part, il ne peut être de discussion publique rationnelle sans la garantie institutionnelle de droits reconnus, et d'autre part, que le telos même de l'agir communicationnel, l'entente, n'est que l'expression d'un projet général de démocratisation de la société, qui s'exprime justement par la revendication de ces droits. En ce sens, ces droits constituent les conditions d'une intersubjectivité authentique, critique, réflexive, donc d'une communication démocratique ouverte et sans contrainte, mais aussi dessinent l'horizon même de la critique démocratique.

Si l'éthique de la discussion constitue un outil très fécond pour penser la dynamique démocratique de création et d'extension des droits, Cohen souligne très justement en quoi elle permet de les fonder, ou tout du moins d'en justifier la visée (Cohen J., Arato A., 1992). Elle spécifie ainsi trois façons de rapporter éthique de la discussion et principes démocratiques. Dans le premier cas, ces principes sont ne constituent qu'un contenu possible d'un accord rationnel. Habermas rejoint ici, bien partiellement, le raisonnement de John Rawls. Dans le second, ils sont déduits de l'éthique de la discussion, parce qu'inscrits dans ses métanormes. Dans le troisième, ils ne sont que présupposés par elle, sans pour autant pouvoir en être déduits.

1. Le premier cas concerne les droits sociaux, impliqués par les exigences de la justice distributive, ainsi que les droits économiques, notamment le droit de propriété. Le rapports de ces droits à l'éthique de la discussion n'est qu'exteme, en aucun cas intrinsèque. Habermas rompt ainsi autant avec l'idéologie libérale, dans sa quête d'une fondation ultime du droit de propriété, mais également socialiste, dans sa tentation de poser la primauté des droits collectifs, sociaux sur les droits individuels, "libéraux". Cette première catégorie de droit ne 
dépend de l'éthique de la discussion que dans la mesure où leur assertion résulte du débat public. Même si on peut être tenté de considérer qu'ils sont, en partie, présupposés dans l'éthique de la discussion comme conditions même de la constitution d'un agent autonome, cette tentation nous parait moins renforcer la légitimité de la revendication de tels droits que, par les confusions conceptuelles auxquelles elle conduit, de l'affaiblir.

2. Cette première catégorie de droits doit être ainsi distinguée d'une seconde, celle des droits de la communication : liberté de parole, d'expression, de réunion, d'association, ... En tant que théorie de la légitimité démocratique, l'éthique de la discussion présuppose, comme l'avait déjà souligné Wellmer de tels droits. En tant qu'ils sont la condition même d'une exercice libre et égale de la critique politique au sein d'cspaces publics, ils sont constitutifs de la discussion elle-même, ils se déduisent de ses méta-principe. Néanmoins l'inscription de ces droits dans la procédure de l'argumentation ne conduit à aucun rousseauisme, elle ne contredit pas la revendication de droits subjectifs.

3. Au contraire, montre l'auteur, l'éthique de la discussion présuppose également, sous une forme différente, cette dernière catégorie de droits. J.Cohen écrit ainsi : "la discussion pratique présuppose des individus autonomes capables de mettre en doute tout consensus donné à partir d'un point de vue défini par des principes" (1990 : 89). Les droits de la subjectivité constituent donc l'une des conditions de la discussion rationnelle car si les participants ne sont pas des agents autonomes, choisissant leurs propres fins, établissant sans contraintes leurs plans de vie, si ils ne sont pas capables d'exercer librement leurs facultés rationnelles, de se mettre à distance de leurs rôles sociaux, des traditions et conventions dans lesquelles ils sont pris, d'en appeler à des principes moraux universalistes, alors il suffirait de s'en remettre, dans l'"argumentation", aux sentences d'un despote éclairé. La liberté négative, l'autonomie subjective définissent ainsi l'identité du sujet de la discussion pratique. Comme l'a souligné A.Wellmer, elle ne se déduit pas de l'éthique de la discussion, à la différence des droits de communication, elle constitue davantage une "méta-condition" de la discussion rationnelle, donc une limite externe au contenu possible d'un accord discursif : on ne saurait, par la discussion, décider d'abolir de tels droits. En effet, reconnaître à l'individu un droit à l'autonomie, c'est lui reconnaître le droit à avoir des droits, donc de participer au débat public.

Ainsi, l'éthique de la discussion exige donc non seulement une validation collective, intersubjective des normes, mais également la capacité même des agents de former des préférences personnelles authentiques, qui pourront ensuite être soumises au test d'universalisation ("principe U"). En ce sens, le consensus que vise la discussion pratique se nourrit d'une pluralité initiale. Il ne la menace pas mais la présuppóse. En tant que conditions d'une intersubjectivité authentique, ces droits appartiennent à l'arrière-plan de l'agir communicationnel, ils font donc partie de ces institutions qui participent à la reproduction du monde vécu. Ces droits, qu'il s'agissent de la liberté de pensée, de parole, d'association, des principes d'inviolabilité et d'autonomie de la personne, garantissent ainsi la préservation et le renouvellement à la fois des traditions culturelles, des formes de solidarités et d'intégration sociales, et des processus de socialisation et de constitution d'identités (Cohen J., Arato A., 1989). 
Plus encore, dans cette perspective renouvelée, toute revendication de droits doit être comprise comme participant à l'approfondissement et à l'extension de la communication, c'est à dire visant l'institutionnalisation de formes discursives plurielles de formation de l'opinion et de la volonté politique. Soit qu'il s'agisse de faire participer à la discussion publique ce qui en étaient exclus, soit qu'il s'agisse de protéger l'autonomie de chacun contre l'Etat ou tout appareil de domination au motif que l'exercice libre et non contraint de sa raison est la condition même de la constitution de sujets authentiques et responsables, capables de se lier et de s'obliger réciproquement, cette revendication, visant au développement de la communication, vise alors celui de la démocratie elle-même. En ce sens, Habermas rejoint G.H Mead (1962 : 327) dans son assimilation entre l'idéal de la démocratie et l'idéal de la communication : " $\mathrm{Si}$ on pouvait dévclopper la communication jusqu'à la rendre parfaite, on obtiendrait alors ce genre de démocratie où l'individu porterait en lui la réaction même qu'il suscite consciemment dans la communauté".

\section{RÉFÉRENCES}

BENHABIB S., 1986, Norm, Critique and Utopia, Columbia University Press.

BENHABIB S., 1990, "Communicative Ethics and Contemporary Controversies in Practical Philosophy", in Benhabib S., Dallmayr F. ed., The Communicative Ethics Controversy, MIT Press.

BERLIN I., 1990, "Deux conceptions de la liberté", in Eloge de la Liberté, Agora.

CHANIAL P., 1994, "Des cercles autopoiétiques aux cercles du langage", in Quaderni $n^{\circ} 22$.

COHEN J., 1990, "Discourse Ethics and Civil Society", in Rasmussen D. ed., Universalism versus Communitarianism, MIT Press.

COHEN J., ARATO A., 1989, "Politics and the Reconstruction of the Concept of Civil Society", in Offe C. et al. Zwischenbetrachtungen, Suhrkamp.

COHEN J., ARATO A., 1992, Civil Society and Political Theory, MIT Press.

HABERMAS J., 1986, Morale et Communication, Cerf.

HABERMAS J., 1987a, Théorie de l'agir communicationnel, t.1, Fayard.

HABERMAS J., 1987b, Théorie de l'agir communicationnel, t.2, Fayard.

HABERMAS J., 1988, "La souveraineté populaire comme procédure", in Lignes $\mathrm{n}^{\circ} 7$.

HABERMAS J., 1992a, "L'Espace Public, 30 ans aprés", in Quaderni n'18.

HABERMAS J., 1992b, De l'Ethique de la Discussion, Cerf.

HABERMAS J., 1993, Faktizität und Geltung, Suhrkamp. 
MEAD G.H., 1962, Mind, Self and Society, University of Chicago Press.

RAWLS J., 1987, Théorie de la Justice, Seuil. WELLMER A., 1989, "Modèles de la liberté dans le monde moderne", in Critique, n'505506.

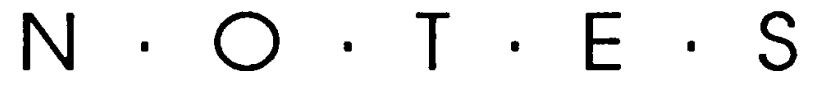

1. L'éthique de la discussion de J.Habermas est formalisée par deux principes (1986: 86-87). Le principe " $D$ " définit dans une logique strictement procédurale l'éthique de la discuusion : "Une norme ne peut prétendre à la validité que sitoutes les personnes qui peuvent être concernées sont d'accord (ou pourraient l'être) en tant que participants à une discussion pratique sur la validité de cette norme". Le principe "U", dit "principe d'universalisation" définit le contenu normatif de cette procédure discursive : "Toute norme valable doit donc satisfaire la condition selon laquelle les conséquences et les effets secondaires qui (de manière prévisible) proviennent du fait que la norme a été universellement observée dans l'intention de satisfaire les intérêts de tout un chacun peuvent être acceptées par toutes les personnes concemées".

2. Sur ces paradoxes de la légitimité de la légalité, voir notre note critique consacrée à N.Luhmann, P.Chanial, 1994. 